ORIGINAL ARTICLE

\title{
Central core disease: clinical, pathological, and genetic features
}

\author{
R M Quinlivan, C R Muller, M Davis, N G Laing, G A Evans, J Dwyer, J Dove, A P Roberts, \\ C A Sewry
}

See end of article for authors' affiliations .....................

Correspondence to: Dr R Quinlivan, Neuromuscular Clinic, Children's Unit, Robert Jones and Agnes Hunt NHS Trust, Oswestry SY10 7AG, UK: Rcmq37@aol.com

Accepted 27 April 2003
Central core disease (CCD) is a dominantly inherited congenital myopathy allelic to malignant hyperthermia $(M H)$ caused by mutations in the RYR1 gene on chromosome 19q13.1. Eleven individuals with RYR1 mutations are described. Four index cases showed features consistent with a congenital myopathy (hypotonia, delayed motor milestones, and skeletal abnormalities including congenital hip dislocation and scoliosis). All four cases and subsequently seven other family members were found to possess novel mutations in the RYRI gene. The degree of disability varied from one clinically normal individual, to another who had never achieved independent ambulation (the only patient with a de novo mutation). Four cases showed a mild reduction in vital capacity, repeated nocturnal polysomnography showed hypoxaemia in one case. A variety of muscle biopsy features were found; central cores were absent in the youngest case, and the biopsy specimens from two others were more suggestive of mini-core myopathy. In all cases missense mutations in exons 101, 102, and 103 of the RYR1 gene on were found. Future laboratory diagnosis of suspected cases and family members will be less invasive and more accurate with DNA analysis. Clinicians, especially paediatricians and orthopaedic surgeons, should be aware of this disorder because of the potential risk of $\mathrm{MH}$.
C entral core disease (CCD) is a dominantly inherited congenital myopathy, first described in 1956 by Shy and Magee. ${ }^{1}$ The incidence is unknown although it is thought to be rare. The diagnosis is made from muscle biopsy by the presence of well defined areas devoid of mitochondria and oxidative enzyme stains, in association with characteristic features. ${ }^{2}$ It is known that CCD is allelic to malignant hyperthermia $(\mathrm{MH})$; indeed the latter may be the presenting feature. Screening of all first degree relatives of affected CCD cases for MH is, therefore, recommended. Furthermore, since most cases will present with orthopaedic deformities it is important that clinicians are made aware of the clinical features, so as to prevent untoward anaesthetic reactions.

Malignant hyperthermia ( $\mathrm{MH})$ is a pharmacogenetic disorder with autosomal dominant inheritance. It is not a disease per se but rather a genetic predisposition in an otherwise normal individual to develop a serious adverse reaction in response to exposure to certain potent inhalational anaesthetics and depolarising muscle relaxants. The most frequent manifestation is isolated masseter muscle rigidity (MMR). ${ }^{3}$ Fever, tachycardia, hypertension, electrolyte disturbances, and rhabdomyolysis leading to acute renal failure, can all occur in varying degrees of severity. ${ }^{45} \mathrm{MH}$ reactions are more prevalent in childhood, with an incidence of 1:15 000 anaesthetics in individuals under 16 years of age, compared with an incidence of 1:50000 to 1:100 000 anaesthetics in adults. ${ }^{5}$ Reactions can be prevented by avoidance of the triggering anaesthetic agents in susceptible individuals.

Current estimates suggest that at least $50 \%$ of $\mathrm{MH}$ individuals will possess a mutation in the gene encoding for the sarcoplasmic reticulum calcium release channel, known as the ryanodine receptor (RYRl). ${ }^{5}$ The majority of families with CCD in which there are enough affected individuals to establish linkage have also been shown to co-segregate to the RYRl gene, found on chromosome $19 \mathrm{q} 13.1$ and comprising 106 exons. ${ }^{6-8}$ The mutated codons giving rise to CCD and $\mathrm{MH}$ are clustered in three regions of the RYRl gene. Regions 1 and 2 reside in the myoplasmic foot domain of the protein. ${ }^{9}$ Region 3 is located in the transmembrane/luminal region of the highly conserved C-terminal domain. ${ }^{9}$ The majority of mutations in $\mathrm{MH}$ and a small number of CCD families were clustered in regions 1 and 2 of the gene. More recently, novel mutations clustering in region 3 of the RYRI in CCD cases have been reported, suggesting a new hot spot in the gene for the disorder. ${ }^{9}{ }^{10}$

We describe the clinical and pathological features of 11 affected individuals from four families presenting at our muscle clinic between 1997 and 2001. These cases highlight the potential difficulties that might occur in establishing the correct diagnosis. All of the index cases were referred to us by paediatric orthopaedic surgeons; some had been assessed in general paediatric clinics for motor delay but had not been investigated to exclude a myopathy. The spectrum of phenotypic expression in terms of severity of disability ranged from no disability to a lack of attainment of independent ambulation. Respiratory insufficiency was found in some cases, a feature not often reported in CCD. Muscle pathology reported in detail elsewhere, ${ }^{11}$ showed a variety of abnormalities which will be discussed, not all of which were diagnostic for CCD although all biopsy specimens contained features that led to the identification of the correct diagnosis. All 11 cases were found to possess novel mutations in region 3 of the RYRl gene.

\section{CASE REPORTS}

Family 1

The index case (table $1, \mathrm{Al}$ ), a white female, presented soon after birth with bilateral congenital dislocation of the hips. At

Abbreviations: CCD, central core disease; FVC, forced vital capacity; IVCT, in vitro contracture test; $M H$, malignant hyperthermia; MMR, masseter muscle spasm; RYR1, ryanodine receptor 
Table 1 Summary of affected individuals, clinical, pathological, and genetic findings

\begin{tabular}{|c|c|c|c|c|c|c|c|c|c|c|}
\hline Patient & Age & MH & Sex & $\mathrm{CDH}$ & Spine & $\begin{array}{l}\text { Facial } \\
\text { weakness }\end{array}$ & $\begin{array}{l}\text { Skeletal } \\
\text { weakness }\end{array}$ & $\begin{array}{l}\text { Respiratory } \\
\text { impairment }\end{array}$ & Biopsy & DNA \\
\hline $\mathrm{Al}$ & $13 \mathrm{~m}$ & $+(?)$ & $\mathrm{F}$ & + & Scoliosis & + & + & - & $\begin{array}{l}\text { Marked increase in } \\
\text { connective tissue, } \\
\text { central cores, } \\
\text { type } 1 \text { fibre uniformity }\end{array}$ & $\begin{array}{l}\text { Ala4940Thr } \\
\text { exon } 103\end{array}$ \\
\hline $\mathrm{A} 2$ & $30 y$ & - & $\mathrm{F}$ & - & Normal & + & - & - & Not done & $\begin{array}{l}\text { Ala4940Thr } \\
\text { exon103 }\end{array}$ \\
\hline B1 & $6 y$ & - & M & - & Scoliosis & + & + & + & $\begin{array}{l}\text { Multiple mini-cores, } \\
\text { type } 1 \text { fibre uniformity }\end{array}$ & $\begin{array}{l}\text { Arg4893Trp } \\
\text { exon } 102\end{array}$ \\
\hline B2 & $16 y$ & - & $\mathrm{F}$ & - & Scoliosis & + & + & + & $\begin{array}{l}\text { Multiple mini-cores, } \\
\text { type } 1 \text { fibre uniformity }\end{array}$ & $\begin{array}{l}\text { Arg4893Trp } \\
\text { exon } 102\end{array}$ \\
\hline B3 & 38 y & - & $\mathrm{F}$ & - & Scoliosis & + & + & + & $\begin{array}{l}\text { Central cores, type } 1 \\
\text { fibre uniformity }\end{array}$ & $\begin{array}{l}\text { Arg4893Trp } \\
\text { exon } 102\end{array}$ \\
\hline $\mathrm{Cl}$ & $3 \mathrm{~m}$ & - & $\mathrm{F}$ & + & Normal & - & + & - & $\begin{array}{l}\text { No cores, type } 1 \\
\text { fibre uniformity }\end{array}$ & $\begin{array}{l}\text { Tyr4864Cys } \\
\text { exon } 102\end{array}$ \\
\hline $\mathrm{C} 2$ & $3 y$ & $+(?)$ & M & - & Lordosis & + & + & - & $\begin{array}{l}\text { Central and } \\
\text { peripheral cores, } \\
\text { type } 1 \text { fibre uniformity }\end{array}$ & $\begin{array}{l}\text { Tyr4864Cys } \\
\text { exon } 102\end{array}$ \\
\hline $\mathrm{C} 3$ & $33 y$ & - & $\mathrm{F}$ & - & Scoliosis & + & + & + & $\begin{array}{l}\text { Multiple mini-cores, } \\
\text { type } 1 \text { fibre } \\
\text { predominance }\end{array}$ & $\begin{array}{l}\text { Tyr4864Cys } \\
\text { exon } 102\end{array}$ \\
\hline $\mathrm{C} 4$ & $42 y$ & - & $\mathrm{F}$ & - & Scoliosis & + & + & - & Not done & $\begin{array}{l}\text { Tyr4864Cys } \\
\text { exon } 102\end{array}$ \\
\hline $\mathrm{C} 5$ & $44 y$ & - & $M$ & - & Normal & - & - & - & Not done & $\begin{array}{l}\text { Tyr4864Cys } \\
\text { exon } 102\end{array}$ \\
\hline D & $11 y$ & - & $M$ & + & Lordosis & + & + & - & $\begin{array}{l}\text { Central cores, type } 1 \\
\text { fibre uniformity }\end{array}$ & $\begin{array}{l}\text { Arg } 4861 \mathrm{His} \\
\text { exon } 101\end{array}$ \\
\hline
\end{tabular}

16 months of age, she underwent open surgical reduction of her hips. During the procedure the orthopaedic surgeon noted pallor of the skeletal musculature and took a sample for biopsy. The child experienced significant hyperthermia of unknown aetiology soon after the anaesthetic and the possibility of a mild malignant hyperthermia reaction was considered, although there was no record of masseter muscle spasm (MMR) or other metabolic abnormality.

On examination at 2 years and 6 months, she had just taken her first independent steps. She was hypotonic with a mild degree of facial weakness. Power in the upper limbs appeared normal but the trunk and hip girdle muscles were weak; she had developed a thoraco-lumbar scoliosis. Her deep tendon reflexes were absent. Serum creatine kinase level was normal, $59 \mathrm{iu} / \mathrm{l}$ (normal range less than $180 \mathrm{iu} / \mathrm{l}$ ). The muscle biopsy taken from the vastus lateralis showed extensive fat and connective tissue, variability in fibre size, and some internal nuclei (fig lA). Oxidative stains showed a uniform pattern, indicating uniformity of fibre typing, with well defined central cores.

Examination of the parents and younger sister showed a mild degree of facial weakness only in the mother (A2). Molecular analysis of the RYRl gene showed a mutation Ala4940Thr in exon 103 in both the child and her mother. However, in the child the aberrant single strand conformation polymorphism band was of the same intensity as the normal band, while in the mother the aberrant band was much fainter than the normal band. The sequencing analysis showed the mutant " $\mathrm{A}$ " peak as slightly larger than the normal " $G$ " peak in the mother (data not shown). We have interpreted these results to suggest that the mother has somatic mosaicism for the gene mutation.

\section{Family 2}

A 5 year old schoolboy of Asian origin (table 1, B1) presented with a scoliosis at $3 \frac{1}{2}$ years of age (fig 2). His mother reported reduced fetal movements and commented that he had been floppy as an infant. He walked at 15 months but at 5 years was unable to hop or jump. He was slow at running compared with his peers and was described as clumsy with a tendency to fall frequently.

On examination, his vital capacity was 0.771 ( $50 \%$ of that expected for his age and height). He had mild facial weakness. Neck flexion weakness was evident but his upper limb power was normal. He had a positive Gower's manoeuvre with moderate hip flexion and extension weakness (MRC 4/5). Deep tendon reflexes were absent. His serum creatine kinase was normal (50 iu/l). Muscle biopsy taken from the vastus lateralis showed a mild degree of fibre size variability. With oxidative enzyme stains, focal areas devoid of stain were seen. These resembled mini-cores in both transverse and longitudinal sections (fig 1, B1).

His elder sister (table 1, B2) was referred to the muscle clinic at 15 years of age. She had been followed up in a paediatric clinic between 2 and 12 years of age because of "poor motor skills". At 12 years of age she developed a scoliosis which required bracing. Her mother reported that she had been floppy as an infant when compared with her clinically normal sister. She was walking by 13 months of age, but had always been poor at motor skills; she climbed stairs only with support and had never been able to run, jump, or hop. On examination, she had reduced muscle tone and deep tendon reflexes. She had mild facial weakness and mild weakness of neck flexion (MRC 4/5); upper limb power was normal. Gower's manoeuvre was positive; there was moderate weakness of all of her hip girdle muscles (MRC $3 / 5$ ). Her thoraco-lumbar scoliosis was controlled by bracing until she was 16 years of age; thereafter no further treatment was required. At 18 years, respiratory insufficiency was noted; forced vital capacity (FVC) was 1.14 litres (32\% of predicted) and nocturnal hypoxaemia was detected on repeated polysomnography studies. Echocardiography was normal. Her serum creatine kinase was less than $20 \mathrm{iu} / \mathrm{l}$. Muscle biopsy taken from the vastus lateralis showed similar features to her brother.

Examination of both parents and the younger sibling revealed the father and child to be clinically normal. The mother (B3), however, showed evidence of mild facial weakness together with weakness of neck flexion and hip 

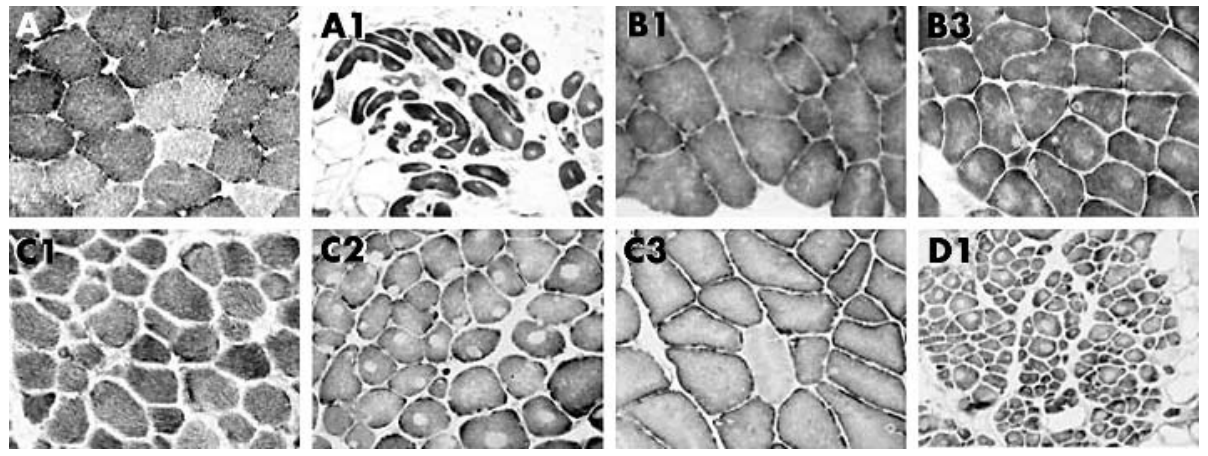

Figure 1 Muscle biopsies stained for NADH-TR. A: normal two fibre pattern. A1: type 1 fibre uniformity (all dark fibres), central cores, and increased adipose and connective tissue. B1 (B2 similar): type 1 fibre uniformity with uneven staining and small core-like areas. B3: type 1fibre uniformity and multiple central and eccentric cores. C1: type 1 fibre uniformity. C2: abundant well defined central and eccentric cores with type 1 fibre uniformity. C3: type 1 fibre predominance, uneven stain, and small eccentric cores. D1: type uniformity, with cores in most fibres, a wide variability in fibre size, and excess adipose tissue.

extension (MRC 4/5). Her upper limb power was normal. She had a positive Gower's manoeuvre but unlike the children, she was able to jump and hop. She had no evidence of any joint contracture or scoliosis. Her deep tendon reflexes were diminished. There was a mild reduction in FVC (2.01 litres; $72 \%$ predicted). Serum creatine kinase was less than $20 \mathrm{iu} / \mathrm{l}$. Muscle biopsy taken from left vastus lateralis showed a spectrum of abnormal oxidative staining with well defined cores. These were single or multiple, peripheral, central, and eccentric in position (fig 1, B3).

Molecular genetic analysis revealed a mutation in RYRl at Arg4893Trp in exon 102 in the mother and two affected children. The mutation segregated with the disease and was not found in the father or younger sibling.

\section{Family 3}

A white female infant (table $1, \mathrm{Cl}$ ), the third child of unrelated parents, presented with bilateral congenital hip dislocation and hypotonia noted from birth. A muscle biopsy was taken from the adductor longus at the time of open hip surgery at 4 months of age. Subsequently her motor milestones were delayed; she rolled from supine to prone at 12 months and sat unsupported at 13 months. She did not walk until 22 months. She had no joint contractures or scoliosis. Deep tendon reflexes were absent. Her muscle biopsy showed a mild variation in fibre size, no internal
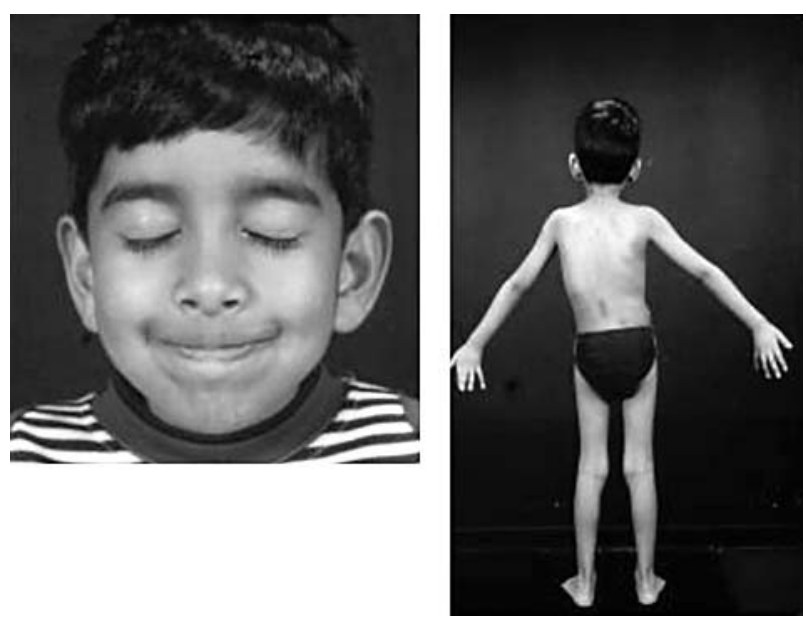

Figure 2 Case B1, showing mild facial weakness with an inability to bury the eyelashes and early onset scoliosis. nuclei or fibrosis. Oxidative stains revealed uniform fibre typing but no cores (fig l, Cl).

Her brother (table 1, C2) was examined in the muscle clinic at the time of his sister's referral. He had been under follow up in a paediatric clinic for developmental delay but a muscle disorder had not been suspected. He sat independently at 11 months but did not walk until 2 years. By 3 years of age, he was unable to run or jump. He had facial weakness and a high arched palate. He had a moderate lumbar lordosis with waddling gait and positive Gower's sign. His deep tendon reflexes were present except for the ankle jerks which were absent. Immediately following routine ear, nose, and throat surgery he developed a pyrexia of unknown origin, although there were no other features to suggest a malignant hyperthermia reaction.

Muscle biopsy taken from the vastus lateralis, showed a mild variation in fibre size, no internal nuclei or fibrosis. Oxidative stains showed uniform fibre typing and abundant peripheral and central cores (fig 1, C2). The father and older sibling were examined and found to be clinically normal.

The mother of the two children (table 1, C3) did not walk independently until 21 months of age. She had never been able to run, jump, or hop and had difficulty climbing stairs. On examination at 33 years of age, she was noted to have facial weakness, and a mild reduction in FVC (2.39 litres, $75 \%$ predicted for height and age). She had weakness of neck flexion, shoulder adduction (MRC 4/5), and moderate weakness of the hip flexors, extensors, adductors, and abductors (MRC 3-4/5). She had a mild thoracic scoliosis. Her deep tendon reflexes were absent. Muscle biopsy from the vastus lateralis showed a mild variation in fibre size with internal nuclei and type 1 fibre predominance. Oxidative enzymes showed small cores in some fibres and unevenness in many fibres, but unlike the children there was no fibre type uniformity (fig 1, C3). She underwent a second muscle biopsy for in vitro contracture testing to exclude $\mathrm{MH}$ which proved negative (Dr Halsall, Leeds, UK).

Figure 3 summarises the family tree; we were informed that the paternal grandfather, who had since died, developed a progressive muscle wasting disease in the sixth decade. His elderly sister was also affected by progressive muscle weakness, apparently beginning in her sixth decade, although we did not have the opportunity to examine her or arrange for molecular genetic testing. We were invited to examine the mother's remaining first degree family members.

The mother's older sister was examined and found to be clinically normal. At her request, she underwent a muscle biopsy for in vitro contracture studies which proved negative for both $\mathrm{MH}$ and CCD. 


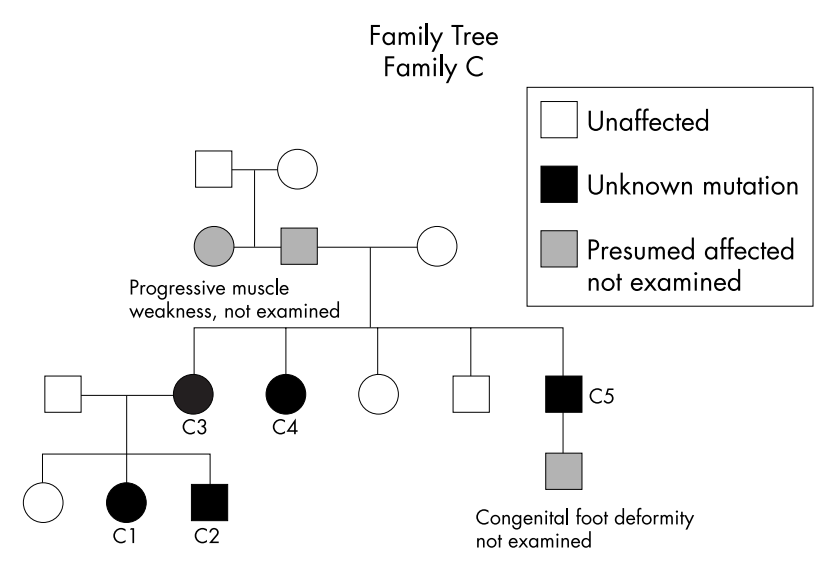

Figure 3 Family tree of family 3.

A second maternal sister (table 1, C4) required scoliosis surgery at 14 years of age. On examination at 42 years of age, she was found to have facial weakness and mild weakness of the hip adductors.

Two maternal brothers were also examined and found to be clinically normal, although one of the brothers (C5) had a son with mild congenital foot deformities (not examined). The maternal grandmother was examined and found to be clinically normal.

Molecular genetic analysis undertaken in all of these individuals showed a Tyr4864Cys mutation in exon 102 of RYR in the two children ( $\mathrm{Cl}$ and $\mathrm{C} 2)$, their mother $(\mathrm{C} 3)$, maternal aunt (C4), and clinically normal uncle (C5).

\section{Family 4}

A white schoolboy (table 1, D1) was referred at 11 years of age with a diagnosis of hereditary spastic paraplegia. The pregnancy was normal; he was born by Ventouse delivery, and no resuscitation was required. He was admitted to the special care baby unit at 2 days of age because of poor feeding. His mother informed us that at the time he was noted to be hypotonic and his legs appeared weak. He acquired head control at the normal time and sat independently at 10 months. Subsequently his motor skills were significantly delayed; he did not roll from supine to prone until 13 months and could not pull himself to stand until 16 months. He was unable to pull himself from a lying to sitting position until 18 months of age. At 3.5 years he walked with a Kaye walker and by 5 years he could walk only with the aid of two sticks; following a period of stability, his gait has become more laboured in recent years and he has never achieved independent aid-free ambulation. He underwent bilateral hip flexor releases at 4 years of age following delayed diagnosis of bilateral congenital hip dislocation. He had no family history of neuromuscular disease.

On examination, his vital capacity was normal at 2.3 litres. He had mild facial weakness and a high arched palate. He was hypotonic and had marked distal joint laxity. There were no joint contractures; he had bilateral scapular winging and a marked lumbar lordosis, but no scoliosis. He walked on his toes with difficulty using two walking sticks for support. His reflexes were present in the upper limbs with facilitation and absent in the lower limbs. His plantar responses were flexor. Sensation for all modalities was normal. Power in the neck flexors, shoulder girdle muscles, and triceps was reduced (MRC 4/5). There was severe muscle weakness in the hip girdle muscles: flexion and abduction (MRC 2-3/5), extension and adduction (MRC $1 / 5)$. Power in the quadriceps was also reduced (MRC 4/5). Examination of both parents was normal.
Investigation showed a normal serum creatine kinase $(40 \mathrm{iu} / \mathrm{l})$ and 12 lead ECG. Hip $x$ rays confirmed high posterior dislocation of the femoral heads bilaterally; $x$ rays and MRI of the whole spine (undertaken at 5 years of age) were normal.

Muscle biopsy taken from the vastus lateralis, showed a variation in fibre size, fibre-type uniformity, and abundant central cores (fig l, D).

Molecular genetic studies showed a heterozygous mutation in RYRl in exon 101: 14582G $>$ A replacing Arg4861 by His. Both parents were tested and found not to possess the mutation, thus representing a spontaneous de novo mutation in this child.

\section{DISCUSSION}

The 11 cases summarised in table 1 show a wide spectrum of phenotypic expression of CCD, ranging from apparently clinically normal (C5) to an inability to attain aid-free independent ambulation (D1). In each of these families the index cases were born with, or developed orthopaedic deformities, namely congenital dislocation of the hips and/ or scoliosis. Facial weakness was a feature in 10 cases. Weakness involving the neck flexors and proximal limb muscles was present in all except two (A2 and C5). The upper limbs were stronger than the lower limbs in all cases. An interesting finding was that the children were more severely affected than their parents. This could be explained by a relative improvement in the condition occurring with advancing age; however, the possibility of anticipation cannot be excluded and long term follow up studies of affected families will be needed to resolve this question.

Respiratory insufficiency is not generally recognised as a feature of CCD and patient B2 might, therefore, be considered to be unusual; in conjunction with her muscle biopsy findings, this could have led to the incorrect diagnosis of mini-core myopathy, a condition with autosomal recessive inheritance, no linkage with $\mathrm{MH}$, but an association with nocturnal hypoventilation. Examination of the mother suggested an autosomal dominant disorder in this family; her muscle biopsy and subsequent unambiguous DNA results confirmed the diagnosis of CCD.

The diagnosis of CCD could also have been missed in family $\mathrm{C}$ in which all of the cases were detected as a consequence of a suspected diagnosis in $\mathrm{Cl}$. However, the muscle biopsy taken from $\mathrm{Cl}$ showed no cores with histochemical stains, the only abnormality being type 1 fibre uniformity. Type 1 fibre uniformity in the absence of cores is a rare but recognised feature in CCD. ${ }^{12}$ Careful examination of the other family members together with a positive muscle biopsy in the mother and sibling led to the correct diagnosis, subsequently confirmed with molecular DNA analysis. The families described in this series highlight the importance of taking a detailed family history and examining the parents of "floppy infants"; CCD may be rare but it is not the only dominantly inherited neuromuscular disorder to present with floppy infant syndrome.

The DNA analysis in our cases, together with those reported recently, ${ }^{10}$ indicate a new hot spot for CCD in region 3 of the RYRl gene, involving exons 95-103. Two of the amino acid substitutions reported here, Arg4861His and Arg4893Trp, have been assayed at the functional level. ${ }^{9}$ The RYRl protein, previously considered to be muscle specific, is expressed at considerable level in B lymphocytes and lymphoblastoid cells. ${ }^{13}$ When calcium release via RYRI was studied in B cells from CCD patients carrying either one of these mutations, a significant spontaneous release of $\mathrm{Ca}^{2+}$ was recorded in the absence of any RYRl trigger. ${ }^{9}$ This may be interpreted as a permanent leakiness of the mutated RYRl, an observation which could well explain the chronic 
muscle weakness in CCD. It is therefore highly likely that these mutations are the genetic cause of the disease.

It is interesting to note that the most severely affected individual in this series (D1) was a spontaneous de novo mutation. Manzur et al reported a severe CCD phenotype in two children from unaffected parents and suggested the possibility of recessive inheritance; DNA analysis was not available to the authors at the time. ${ }^{14}$ Recently, however, DNA analysis has shown such sporadic cases to possess heterozygote mutations in the RYRl gene, and has suggested that de novo mutations are relatively common among CCD cases. ${ }^{10}$

Testing for MH involves an in vitro contracture test (IVCT) developed by the European and North American malignant hyperthermia groups. ${ }^{15} 16$ The test requires a large fresh muscle biopsy and is not easily performed in children. It is based on the tendency of MH muscle to be abnormally sensitive to stimuli that induce sarcoplasmic reticulum calcium release. The degree of muscle contraction is measured on flooding or gradually increasing concentrations of halothane or caffeine. In general, the correlation between the results of the two tests is good and the test shows a high sensitivity (99\%) and specificity (93\%). ${ }^{17}$ The results can, however, be equivocal and discrepancies, both false positive and false negative results, compared to DNA results have been reported. ${ }^{18}$ Patient C3 had a negative IVCT, but was subsequently shown to possess a mutation in the RYRl gene. The contradictory nature of her results could represent a false negative IVCT, or alternatively, it could be that mutations in this part of the RYRl gene are less likely to be associated with $\mathrm{MH}$. None of our patients experienced a fulminant $\mathrm{MH}$ reaction although this would not necessarily exclude $\mathrm{MH}$, since about one half of all MH reactions can be preceded by up to 13 previously uneventful general anaesthetics. ${ }^{5}$ It has been suggested that mild episodes of MH could go unnoticed, especially if anaesthesia is conducted without end tidal $\mathrm{CO}_{2}$ monitoring. Prior to the diagnosis of CCD, two patients, Al and $\mathrm{C} 2$, developed high fever immediately following general anaesthesia. Even though there was no evidence of MMR or metabolic disturbance in either case and although we did not have information relating to the perioperative monitoring, the possibility of mild $\mathrm{MH}$ cannot be completely excluded. Until further IVCT studies are undertaken in individuals with mutations in this region of the RYRl gene, all confirmed cases of CCD should be considered to be at risk for MH.

In summary, CCD is thought to be rare but because of the variability in phenotypic expression and its non-progressive nature, it seems likely that as yet undiagnosed cases exist within the general population. Paediatricians and orthopaedic surgeons should be aware of the condition because of the genetic implications and the close association with $\mathrm{MH}$, especially as new cases are likely to present to those specialty clinics. The diagnosis should be considered in any child or young adult presenting with weakness, hypotonia in infancy, and/or skeletal abnormalities. A careful family history and examination of the parents may be useful and should be done routinely for investigation of all floppy infants. The diagnosis of CCD can be confirmed by muscle biopsy (creatine kinase and electromyogram may be normal), but exceptions will include cases showing only type 1 fibre predominance, or multiple small cores resembling mini-core myopathy especially in the very young. The finding of a new hot spot for CCD at the C-terminal RYRI receptor gene, with mutations occurring in exons 95-103, is likely to resolve these difficulties and improve diagnostic accuracy in the future.

\section{Authors' affiliations}

R M Quinlivan, G A Evans, A P Roberts, C A Sewry, Neuromuscular Clinic and Departments of Pathology and Radiology, The Robert Jones and Agnes Hunt District and Orthopaedic NHS Trust, Oswestry, Shropshire SY10 7AG, UK

C R Muller, Department of Human Genetics, University of Wuerzberg, Wuerzberg 97074, Germany

M Davis, Department of Anatomical Pathology, Royal Perth Hospital, Perth, Australia

N G Laing, Centre for Neuromuscular and Neurological Disorders, University of Western Australia, Australian Neuromuscular Research Institute, QE11 Medical Centre, Perth, Australia

J Dwyer, J Dove, Department of Paediatric Orthopaedics and Paediatrics, North Staffordshire Royal Infirmary, Stoke on Trent, UK

\section{REFERENCES}

1 Shy GM, Magee KR. A new congenital non-progressive myopathy. Brain 1956;79:610-21.

2 Hayashi K, Miller G, Brownell AK. Central core disease: ultrastructure of the sarcoplasmic reticulum and T-tubules. Muscle Nerve 1989;12:95-102.

3 Kosko JR, Brandom BW, Chan KH. Masseter spasm and malignant hyperthermia: a retrospective review of a hospital-based paediatric otolaryngology practice. Int J Pediatr Otorhinolaryngol 1992;23:45-50.

4 King JO, Denborough MA. Malignant hyperpyrexia in Australia and New Zealand. Med J Aust 1973;1:525-8.

5 Britt BA. Malignant hyperthermia-a review. In: Schonbaum E, Lomax P, eds. Thermoregulation: pathology, pharmacology and therapy. New York: Pergamon Press, 1991:179-292.

6 MacLennon DH, Duff C, Zorzato F, et al. Ryanodine receptor gene is a candidate for predisposition to malignant hyperthermia. Nature 1990;343:559-61.

7 Loke J, MacLennan DH. Malignant hyperthermia and central core disease, disorders of $\mathrm{Ca}^{2+}$ release channels. Am J Med 1998;104:470-86.

8 McCarthy TV, Quane KA, Lynch PJ. Ryanodine receptor mutations in malignant hyperthermia and central core disease. Hum Mutat 2000;15:410-17.

9 Tilgen N, Zorzato F, Halliger-Keller B, et al. Identification of four novel mutations in the $\mathrm{C}$-terminal membrane spanning domain of the ryanodine receptor 1: association with central core disease and alteration of calcium homeostasis. Hum Mol Genet 2001;10:1-9.

10 Monnier N, Romero NB, Lerale J, et al. Familial and sporadic forms of central core disease are associated with mutations in the C-terminal domain of the skeletal muscle ryanodine receptor. Hum Mol Genet 2001;10:2581-92.

11 Sewry CA, Muller C, Davis M, et al. The spectrum of pathology in central core disease. Neuromuscular Disorders 2002;12:930-8.

12 Tojo M, Ozawa M, Nonaka I. Central core disease with uniform type 1 fibres in one family. Brain Develop 2000;22:262-4.

13 Girard T, Cavagna D, Padovan E, et al. B-lymphocytes from malignant hyperthermia-susceptible patients have an increased sensitivity to skeletal muscle ryanodine receptor activators. J Biol Chem 2001;276:48077-82.

14 Manzur AY, Sewry CA, Ziprin J, et al. A severe clinical and pathological variant of central core disease with possible autosomal recessive inheritance. Neuromusc Dis 1998:8:467-73.

15 European MH group. A protocol for the investigation of malignant hyperpyrexia (MH) susceptibility. Br J Anaesth 1984;56:1267-9.

16 Larach MG. Standardisation of the caffeine halothane muscle contracture test. Anaesth Analg 1989;69:511-15.

17 Jurkatt-Rott K, McCarthy T, Lehmann-Horn F. Genetics and pathogenesis of malignant hyperthermia. Muscle Nerve 2000;23:4-17.

18 MacLennon D. Discordance between phenotype and genotype in malignant hyperthermia. Curr Opinion Neurol 1995;8:397-401. 